\title{
Prevalence of chronic idiopathic musculoskeletal pain and headache in adolescence: a population based Norwegian study
}

\author{
Gry B Hoftun ${ }^{3 *}$, Pål R Romundstad ${ }^{1}$, John-Anker Zwart ${ }^{2}$, Marite Rygg ${ }^{3}$ \\ From 2011 Pediatric Rheumatology Symposium sponsored by the American College of Rheumatology \\ Miami, FL, USA. 2-5 June 2011
}

\section{Purpose}

The aim of this study was to determine the prevalence of chronic idiopathic musculoskeletal pain and headache among adolescents in relation to age and gender.

\section{Methods}

A population-based, cross-sectional study was carried out in Nord-Trøndelag county, Norway, during the years 2006-2008. All adolescents aged 12-19 years were invited to participate in the youth part of the Nord-Trøndelag Health Study ["Helseundersøkelsen i Nord-Trøndelag (HUNT)"]. Response rate was 78\% (8200 responders out of 10.485 invited). The adolescents completed a comprehensive questionnaire including questions about whether they had experienced any headaches and/or any musculoskeletal pain during the last three months, not related to any known disease or injury. They were asked to indicate the location of pain (neck/shoulders, upper back, lower back/buttocks, chest, upper and/or lower extremities). The participants were also asked to indicate how often they had experienced pain in these areas (seldom or never, once a month, once a week, more than once a week, almost every day). The final study population, with complete pain questionnaires, consisted of 7373 adolescents aged 13-18 years.

\section{Results}

Pain at least once a week during the last three months was reported by $44 \%$ ( $95 \%$ CI $43-45 \%$ ), including $54 \%$ (95\% CI $53-56 \%$ ) of the girls and 34\% (95\% CI 33-36\%) of the boys. The prevalence of headache at least once a week during the last three months was $22 \%$ (95\% CI 21-23\%), including

${ }^{3}$ Norwegian University of Science and Technology, Faculty of Medicine/St. Olav's Hospital, Trondheim, Norway

Full list of author information is available at the end of the article
$30 \%$ (95\% CI $29-31 \%$ ) of the girls and $14 \%$ (95\% CI $13-$ $15 \%)$ of the boys, and for musculoskeletal pain 33\% (95\% CI $32-34 \%$ ), girls $40 \%$ (95\% CI $38-42 \%$ ) and boys $27 \%$ (95\% CI 25-28\%). Pain in the neck/shoulder region was the most commonly affected musculoskeletal region, reported by $17 \%$ (22\% of the girls and $12 \%$ of the boys). Pain prevalence was higher in girls than in boys in all age groups and for all pain categories. The prevalence of pain increased with age in most pain categories and locations, except for pain in upper and lower extremities.

\section{Conclusion}

Chronic pain, defined as pain at least once a week present for at least three months, is common among Norwegian adolescents, with higher prevalence in girls than boys. Musculoskeletal pain shows the highest prevalence, and is reported by one third of the study population.

\section{Disclosure}

Gry B Hoftun: None; Pål R Romundstad: None; JohnAnker Zwart: None; Marite Rygg: None.

\section{Author details}

${ }^{1}$ Norwegian University of Science and Technology, Faculty of Medicine, Trondheim, Norway. ${ }^{2}$ Norwegian University of Science and Technology, Faculty of Medicine/Oslo University Hospital, Ullevål, and University of Oslo, Trondheim/Oslo, Norway. ${ }^{3}$ Norwegian University of Science and Technology, Faculty of Medicine/St.Olav's Hospital, Trondheim, Norway.

Published: 13 July 2012

doi:10.1186/1546-0096-10-S1-A77

Cite this article as: Hoftun et al:: Prevalence of chronic idiopathic musculoskeletal pain and headache in adolescence: a population based Norwegian study. Pediatric Rheumatology 2012 10(Suppl 1):A77.

\section{()

(C) 2012 Hoftun et al; licensee BioMed Central Ltd. This is an Open Access article distributed under the terms of the Creative Commons Attribution License (http://creativecommons.org/licenses/by/2.0), which permits unrestricted use, distribution, and reproduction in any medium, provided the original work is properly cited. 